\title{
Is Transvaginal Sonograpy Useful in Women's Health Care Programs?
}

\section{Hiroyuki Kuramoto*}

Department of Cancer Detection for Females, The Kanagawa Health Service Association, Graduate School of Medical Sciences, Kitasato University and Meiji Yasuda Life Foundation of Health and Welfare, Japan

\begin{abstract}
Objective: The role and efficacy of trans-vaginal ultra-sonography (TVS) in women's health care programs were evaluated.

Methods and materials: The subjects were 1,000 consecutive women who received a health care program with TVS. Gynecologic abnormalities detected by TVS were compared with those from a bimanual pelvic exam.

Results: 1) The incidence of Gynecologic abnormalities found by TVS was $24.5 \%$, whereas that by pelvic exam was $13.6 \%$. 2) The incidence of detected uterine myoma by TVS was $20.4 \%$, whereas that by the exam was $8.3 \%$. When compared with myoma size, myomas of $<2 \mathrm{~cm}, 2-3 \mathrm{~cm}, 3-5 \mathrm{~cm}, 5-7 \mathrm{~cm}$ and $\geq 7 \mathrm{~cm}$ in diameter were distributed in $26.3 \%$, $22.0 \%, 27.3 \%, 13.2 \%$ and $11.2 \%$, respectively. Among those found by TVS, $1.9 \%, 15.6 \%, 46.4 \%, 96.3 \%$ and $100 \%$ were detectable by pelvic exam, respectively. 3) Incidences of both ovaries, right ovary and left ovary visible and both ovaries invisible by TVS were $26.0 \%, 15.4 \%, 14.6 \%$ and $44.1 \%$, respectively. When compared with the total number of ovaries, $41 \%$ of ovaries were only visualized by TVS. 4) Ovarian tumors were detected in $2.0 \%$ by TVS, whereas in $1.0 \%$ by pelvic exam.

Conclusions: TVS is more sensitive to detect Gynecologic abnormalities than pelvic exams. The high sensitivity of TVS allows it to find smaller myoma nodules and thus increases the number of gynecologically abnormal women. In contrast, ovaries are not visualized in the majority of cases.
\end{abstract}

Keywords: Trans-vaginal sonography; Pelvic exam; Health care

\section{Introduction}

Transvaginal ultrasonography (TVS) is a convenient method to detect morphological abnormalities in the Obstetric and Gynecologic fields, and may substitute for a bimanual pelvic examination. Detailed analysis of the effectiveness of TVS, however, has not yet been done, except for its role in the early detection of ovarian cancer [1-6].

The annual health care program i.e. "Ningen (Human) Dock" in Japan is nowadays a popular method for early detection of diseases, and TVS is commonly used to detect Gynecologic abnormalities in health care programs for women without concrete evidence of its usefulness. The present study evaluated the role and efficacy of TVS in a women's health care program.

\section{Materials and Methods}

\section{Materials}

The subjects were 1,029 consecutive healthy women who gave informed consent and who entered a health care program i.e. "human dock" at Shinjuku Medical Center, Meiji Yasuda Life Foundation of Health and Welfare between July 16, 2010 and May 25, 2012. Their mean age \pm SD was $48.5 \pm 10.8$ years old. Repeaters whose health care with TVS had been performed by the author during the period were excluded. The screening programs are based on company regulations or individual applications.

\section{Methods}

The Gynecologic aspect of the program includes history taking, a Pap smear, a bimanual pelvic examination and TVS in that order in the health care program. The pelvic exam was not repeated after TVS to avoid any effect of TVS findings. Women's history was taken by nurses in charge who had answers to previously prepared questionnaires, and the rest of the program was done by the author, who is certified an
Obstetrician and Gynecologist licensed by Japan Society of Obstetrics and Gynecology, and a Gynecologic Oncologist licensed by Japan Society of Gynecologic Oncology. Routine operating time of the program is 5 to 6 minutes excluding history taking. The results of Pap smears were excluded from this study.

1) Categorization of abnormalities detected and suggestions for the recipients

The results of gynecologic abnormalities found in the program were categorized, and personalized instructions and suggestions were given to the recipients of the program in the following categories:
A: no abnormal findings
B: minimal abnormality found, but not disturbing for daily life
$\mathrm{C} 1$ : re-examination in one month
C3: follow-up and re-examination in 3 months
C6: follow-up and re-examination in 6 months
C12: follow-up and re-examination in 12 months
D1: treatment needed
D2: detailed exam needed
E: treatment and care should be continued

*Corresponding author: Hiroyuki Kuramoto, Department of Cancer Detection for females, The Kanagawa Health Service Association, Kitasato University and Meiji Yasuda Life Foundation of Health and Welfare, 58 Nihon-Ohdori Naka-ku Yokohama-shi, Kanagawa, 231-0021, Japan, Tel: +81-45-461-8645; E-mail: Kuramoto@yobouigaku-kanagawa.or.jp

Received November 18, 2013; Accepted November 29, 2013; Published December 02, 2013

Citation: Kuramoto H (2013) Is Transvaginal Sonograpy Useful in Women's Health Care Programs? Health Care Current Reviews 1: 110. doi: 10.4172/2375-4273.1000110

Copyright: $\odot 2013$ Kuramoto $\mathrm{H}$. This is an open-access article distributed under the terms of the Creative Commons Attribution License, which permits unrestricted use, distribution, and reproduction in any medium, provided the original author and source are credited. 
Citation: Kuramoto H (2013) Is Transvaginal Sonograpy Useful in Women's Health Care Programs? Health Care Current Reviews 1: 110. doi: 10.4172/2375-4273.1000110

Page 2 of 5

\section{2) Transvaginal ultrasonography}

A Sonovista-C 3000 (Mochida-Siemens Medical Systems Co. Ltd, Tokyo) was used for transvaginal ultrasonography, using a mechanical sector probe with a semi-spherical top edge that covered $220^{\circ}$ fields in the $2 \mathrm{D}$ mode. The standard wavelength for the procedure was 7.5 $\mathrm{MHz}$. Two photos were taken routinely; one measuring the widths of the uterine corpus and endometrium and one in each half of the other, either the right or left ovary (Figures 1 and 2). The area with an iliac vein in the pelvic side wall was photographed, if ovaries were not visualized.

An ovarian cystic mass was defined to be one with a diameter of $2.5 \mathrm{~cm}$ or greater and ovarian tumors were grouped into six types, following the criteria of the Japan Society of Ultrasonics in Medicine [7]. In brief,

Type I: cystic without echo in content

Type II: cystic with some echo

Type III: mixed pattern with central or peripheral solid echo, smooth in border

Type IV: mixed pattern, prominently cystic, with irregular solid or uneven lobular border

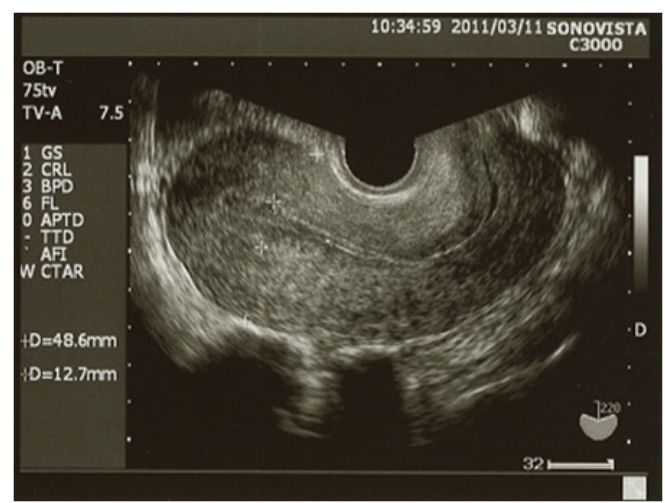

Figure 1: Typical TVS findings of the uterus and the ovulatory phase of the endometrium.

Slight adenomyosis is seen in the posterior wall of the corpus in a 40 year-old woman with algomenorrhea.

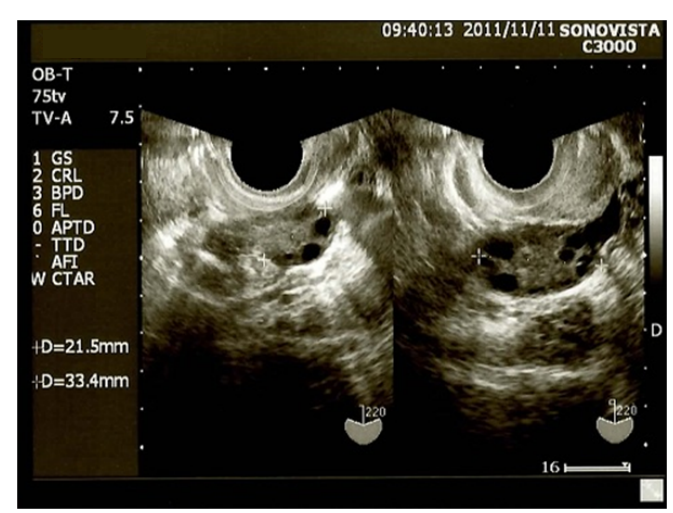

Figure 2: Typical TVS findings of the ovaries; right ovary in the left half and left ovary in the right half.

Follicles are developing well in a 39 year-old woman.
Type V: mixed pattern, prominently solid

Type VI: solid pattern

3) Levels of suggestions for uterine myomas and ovarian tumors

The basic instructions for women who had uterine myomas or ovarian tumors, etc. were as follows, depending on the size and type on TVS.

Uterine myoma

$<3 \mathrm{~cm}$ : B, excluding that with $<2 \mathrm{~cm}$

3-5 cm: $\mathrm{C} 12$

$5-7 \mathrm{~cm}: \mathrm{C} 6$

$\geq 7 \mathrm{~cm}: \mathrm{C} 3$

Bigger than fist size: D2

Suggestions could be modified according to symptoms such as hypermenorrhea

Ovarian tumors

Simple cysts (Type I and II)

$2.5-3 \mathrm{~cm}: \mathrm{B}$

$3-5 \mathrm{~cm}: \mathrm{C} 6$

$5 \mathrm{~cm}$ : detailed exam

Type III-VI: detailed exam

Endometrial thickness $\geq 5 \mathrm{~mm}$ in postmenopausal woman: D2

4) Statistical analysis

Statistical analysis was performed using Excel Statistics 2010 software program for Windows ${ }^{\circledR}$ (SSRI, Tokyo). Fisher's test was used to compare the results between TVS and pelvic exam.

\section{Results}

Twenty-two and six women had undergone hysterectomy with or without salpingo-oophorectomy, and unilateral salpingooophorectomy, respectively. Therefore, the former were excluded from the analysis of uterine myoma and both were excluded from that of the ovary. Consequently, the total number of women without surgery was 1,001 .

\section{Comparison of abnormality incidences between TVS and the pelvic exam}

The abnormal findings found by TVS were 252 out of 1,029 , or $24.5 \%$, and those by the pelvic exam were 140 , or $13.6 \%(\mathrm{p}<0.01$, Figure $3)$.

\section{Incidence of uterine myoma detection}

Two hundred and five women out of 1,007 , or $20.4 \%$, were found to have uterine myoma by TVS, but only $8.3 \%$ were found by the pelvic exam $(p<0.0000$, Figure 4$)$. Forty-one percent of those found by TVS were only detected by the pelvic exam. Women with myomas less than $2 \mathrm{~cm}, 2-3 \mathrm{~cm}, 3-5 \mathrm{~cm}$ (Figure 5), $5-7 \mathrm{~cm}$, and $7 \mathrm{~cm}$ or greater in the longer diameter were 54 (26.3\%), 45 (22.0\%), 56 (27.3\%), 27 (13.2\%) and $23(11.2 \%)$, respectively, when compared with myoma size on TVS. When compared with the pelvic exam, $1.9 \%, 15.6 \%, 46.4 \%, 96.3 \%$ and $100 \%$ were detected by the exam, respectively, and the findings of palpable cases depending on their sizes are listed in Table 1.

\section{Incidences of visualization of the ovaries by TVS}

Out of 1,001 women without pelvic surgery, cases with both ovaries visible were $26.0 \%$, those with right ovaries visible were $15.4 \%$, 
Citation: Kuramoto H (2013) Is Transvaginal Sonograpy Useful in Women's Health Care Programs? Health Care Current Reviews 1: 110. doi: 10.4172/2375-4273.1000110

Page 3 of 5

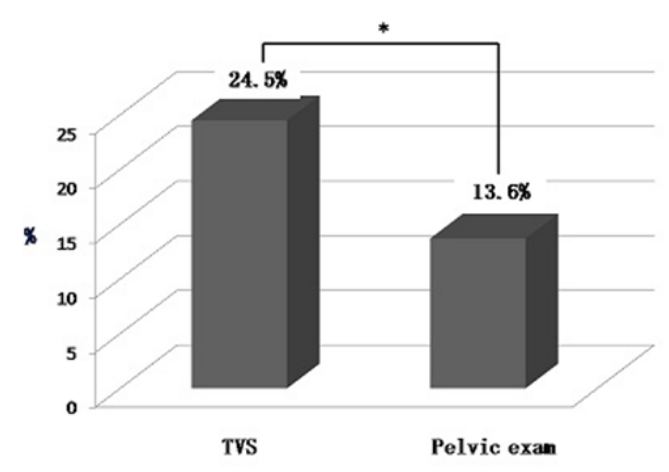

Figure 3: Incidences of detected Gynecologic abnormalities - Comparison between TVS and the bimanual pelvic exam $(n=1029)$.

TVS was more sensitive to detect abnormalities than the pelvic exam. *Fisher's exact probability test $p<0.01$.

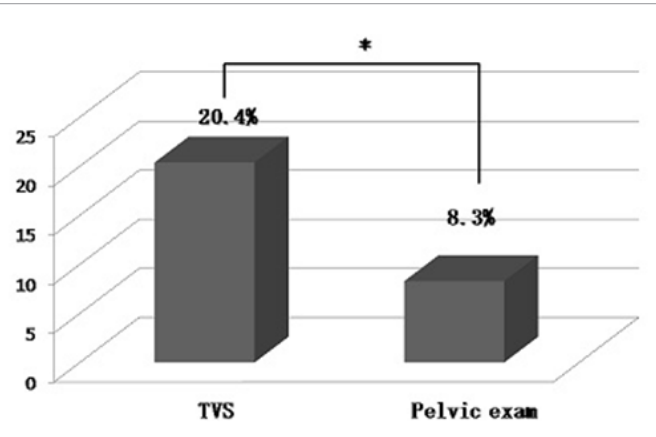

Figure 4: Incidences of detected uterine myomas - Comparison between TVS and the pelvic exam.

TVS was more sensitive to detect abnormalities than the pelvic exam. *Fisher's test $\mathrm{P}<0.0000$

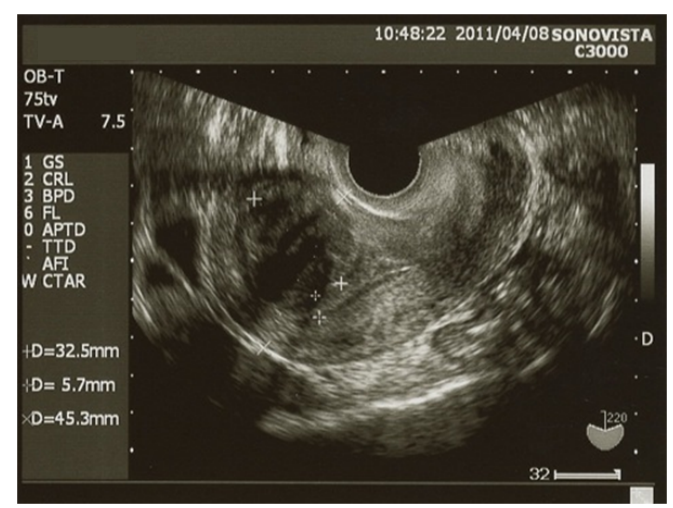

Figure 5: A small uterine myoma of $32.5 \mathrm{~mm}$ in diameter in a 41 year-old woman.

those with left ovaries visible were $14.6 \%$ and those with both ovaries invisible were $44.1 \%$.

Eight-hundred and twenty ovaries, or $41 \%$, were visible by TVS, when counting the total number of ovaries i.e. 2,002, whereas $59 \%$ were invisible. When analyzed with coincidental uterine myoma, the incidence of invisible ovaries was $71.2 \%$ in those $(n=212)$ with myoma $\geq 3 \mathrm{~cm}$ in size and $73 \%$ in those $(\mathrm{n}=100)$ with myoma $\geq 5 \mathrm{~cm}$, both of them were significantly higher than those without myoma $(\mathrm{p}<0.0005$ and $\mathrm{p}<0.0063$, respectively. Figure 6).

\section{Incidence of ovarian tumor detection}

Ovarian tumors by TVS were detected in 20 , or $2.0 \%$, of 1001 cases, whereas those detected by the pelvic exam were 10 , or $1.0 \%$. There was no significant difference between the two (Fisher's test). Ten women who were not detected by the pelvic exam had tumors less than 43.8 $\mathrm{mm}$ in diameter. Types of ovarian tumors and their categories were Type I in 11 cases (B;2, C3; 1, C6; 5, C12; 1 and D2; 2), Type II in 4 (C3; 1 and C6; 3), Type V in 1 (D2) and Type VI in 4 (C6; 2 and D2; 2, Figure 7), and no malignant tumors were detected, including the cases under research.

\section{Miscellaneous gynecologic diseases detected}

Gynecologic diseases ( $n=140,147$ lesions) found by history taking and pelvic exam and their categories are listed in Table 2. Miscellaneous diseases other than uterine myomas and ovarian tumors found by

\begin{tabular}{|l|l|l|}
\hline $\begin{array}{l}\text { Diameter of } \\
\text { myoma }\end{array}$ & $\begin{array}{l}\text { Abnormality by pelvic } \\
\text { exam \% (n) }\end{array}$ & Findings of pelvic exam \\
\hline$<2 \mathrm{~cm}$ & $1.9 \%(54)$ & Irregular surface of uterus $(\mathrm{n}=1)$ \\
\hline $3-2 \mathrm{~cm}$ & $15.6 \%(45)$ & Multiple $(\mathrm{n}=5)$, irregular surface $(\mathrm{n}=1)$ \\
\hline $5-3 \mathrm{~cm}$ & $46.4 \%(56)$ & (small) goose egg size \\
\hline $7-5 \mathrm{~cm}$ & $96.3 \%(27)$ & Small-large goose egg size \\
\hline$>7 \mathrm{~cm}$ & $100.0 \%(23)$ & \\
\hline
\end{tabular}

Table1: Incidences of abnormalities found by the pelvicexam and the sizes of uterine myomas on TVS ( $\underline{n}$ 205).

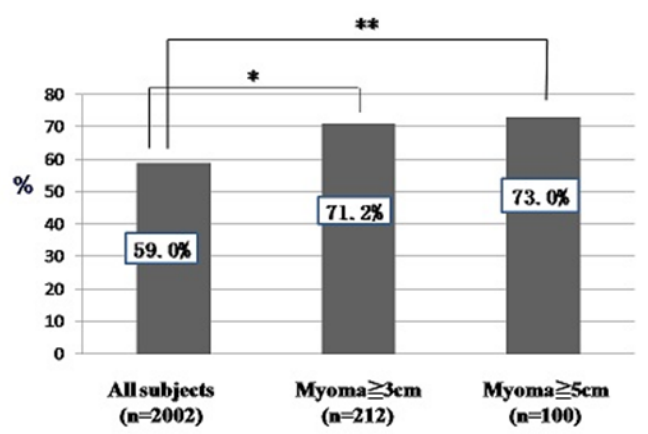

Figure 6: Incidences of invisible ovaries on TVS - Comparison between al subjects and those with coincidental uterine myoma.

The incidences were higher in women with myoma than all subjects. *Fisher's test, both side $p<0.0005,{ }^{* *} p<0.0063$

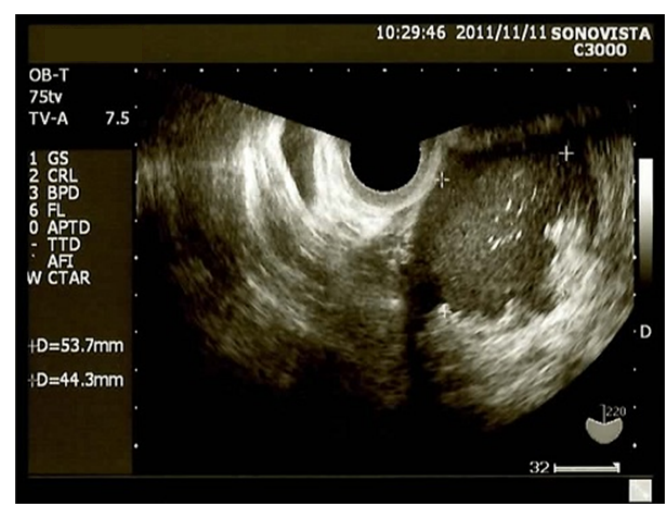

Figure 7: Ovarian tumor, Type $\mathrm{VI}$, of $53.7 \times 44.3 \mathrm{~mm}$ in a 58 year-old woman. 
Citation: Kuramoto H (2013) Is Transvaginal Sonograpy Useful in Women's Health Care Programs? Health Care Current Reviews 1: 110. doi: 10.4172/2375-4273.1000110

TVS ( $\mathrm{n}=33$ ) were fluid retention in the uterine cavity (Figure 8) in 19 cases, or $1.9 \%$ of 1,001 cases, adenomyosis (Figure 1) in 7 , or $0.7 \%$, endometrial thickness ( $\geq 5 \mathrm{~mm}$ ) in postmenopausal women (Figure 9) in 4 , or $0.4 \%$ and cervical cyst (Figure 10 ) in 3, or $0.3 \%$. On the contrary, the endometrium was not visualized in $2.6 \%$.

\section{Discussion}

Gynecologic abnormalities were found by pelvic exam in $13.6 \%$,

\begin{tabular}{|l|l|}
\hline Vulva & Adnexa \\
\hline Vulvitis 1(E) & Ovarian tumor 10(C3;1, C6;4, D2;5) \\
\hline Balthorin cyst 3(C12) & Functional \\
\hline Condyloma 1(D2) & Algomenorrhea 2(B) \\
\hline Leukoplakia 1(C12) & Abnormal Menses 4(D2; 2, B;2) \\
\hline Tumor 2(C12) & Metrorrhagia 3(C3; 1, D2;2) \\
\hline Vagina & Ovarian dysfunction 4(D1;1, D2;!, E;2) \\
\hline Vaginitis 12(D1;9, B;3) & Others \\
\hline Cervix & Deformed cervix 1(C3) \\
\hline Endocervical polup 15(B;10, D1;5) & \\
\hline Portio polyp 2(B) & \\
\hline Corpus & \\
\hline $\begin{array}{l}\text { Myoma 83(B;4, C6;22, C12:42, D1;1, } \\
\text { D2;10, E;4) }\end{array}$ & \\
\hline Myomatous uterus 1(C12) & \\
\hline Descendent uterus 1(B) & \\
\hline
\end{tabular}

Table 2: Gynecologic diseases detected by pelvicexam and their categories for instruction.

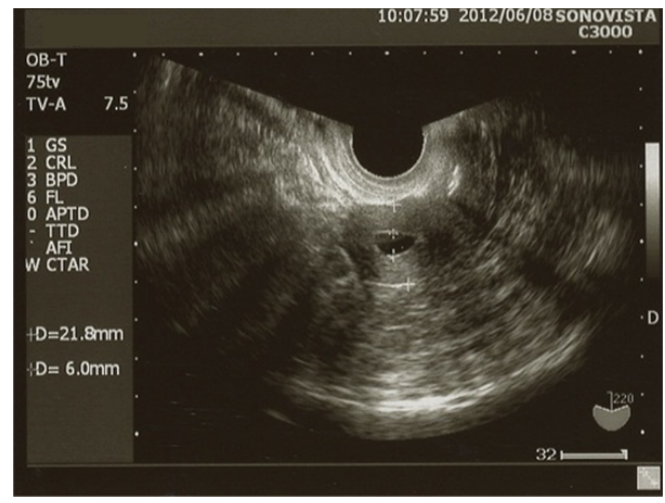

Figure 8: Retention of fluid in the endometrial cavity, $6.0 \mathrm{~mm}$ thick, in a 65 year-old woman.

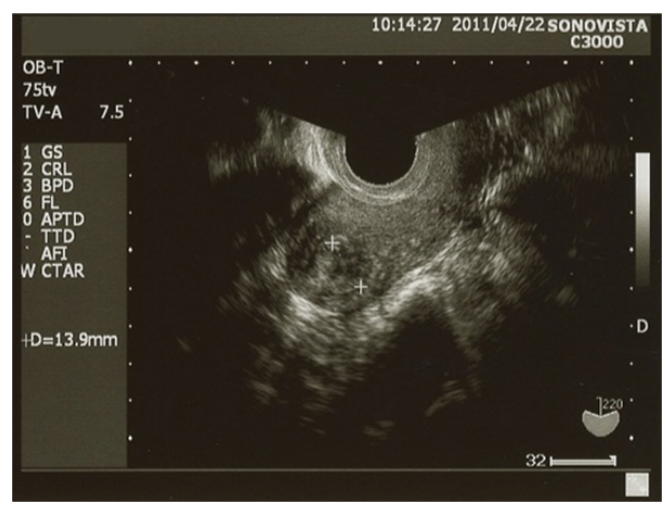

Figure 9: Thick and irregular endometrium in a 44 year-old woman.

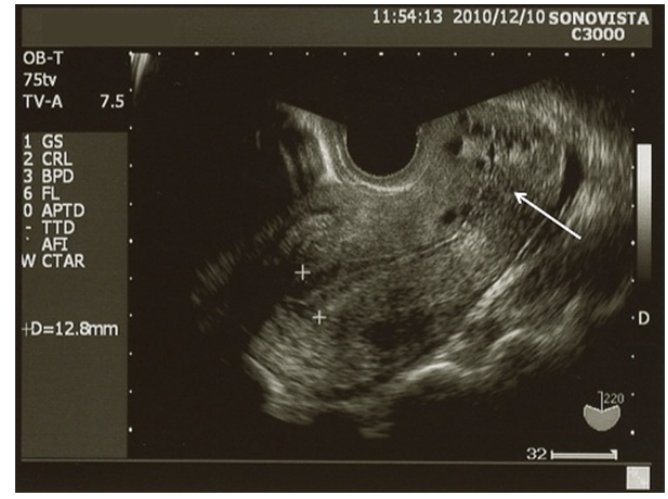

Figure 10: Multiple cysts located in the cervix (arrow) in a 48 year-old woman

whereas in our previous report it was $7.9 \%$ [8]. The incidence in the present report may be due to the effect of a careful pelvic exam that conflicted with the sensitivity of TVS. In contrast, at $24.5 \%$, the incidence by TVS was extremely high compared with that of the pelvic exam, and means one quarter of basically healthy women have gynecologic diseases from which they may suffer a disease, even though these are neglected abnormalities. The high incidence of abnormality by TVS is mainly due to the high sensitivity of detecting uterine myoma i.e. $20.4 \%$ of the program recipients. The incidence of myoma would decrease to $10.5 \%$ if we ignore myomas $<3 \mathrm{~cm}$ in size. Therefore, it is necessary to exclude myomas $<3 \mathrm{~cm}$ in size, if there are no symptoms. The bimanual pelvic exam did not detect uterine myomas of $<3 \mathrm{~cm}$ with some exceptions, and approximately half of those of 3-5 cm. These small myomas have no clinical importance unless there are symptoms. Therefore, the pelvic exam is a reasonable method of detecting gynecologic abnormalities.

The majority (59\%) of ovaries were not visualized by TVS in the present study. In contrast, van Negell Jr et al. [1] reported that at least one ovary was not seen in $16 \%$, and spent a minimum time of 5 minutes to identify each ovary [2]. Spending more than ten minutes to identify ovaries, however, may be too long for healthy women. They $[1,2]$ also defined that the ovary was regarded as negative for abnormalities, if it was not visualized. It should be defined as undetermined, since TVS is one of visual diagnostic methods. The sensitivity and specificity of TVS to define the ovaries as normal is very low. Therefore, it may not be recommended to perform TVS alone without a bimanual pelvic exam. Hiramoto et al. [9] reported that ovarian tumors were detected by TVS in $2.96-4.27 \%$, whereas in our experience it was $2 \%$. The ovarian tumors that were detected by TVS and not by the pelvic exam were all plain cystic masses of $<4.4 \mathrm{~cm}$ in diameter.

Sato et al. [10] reported that using TVS for ovarian cancer screening the incidence of detected ovarian cancer was $0.04 \%$ among more than 50,000 women screened and $77.3 \%$ of them were in stage I, so the TVS screening may increase the chance for early diagnosis and reduce the mortality of ovarian cancer. Kobayashi et al. [11] reported that the ovarian cancer risk was elevated significantly among patients with ovarian endometrioma found by transabdominal and/ or transvaginal ultrasound in the Cohort Study on Endometriosis and Ovarian Cancer Programme, and increased with increasing age at ovarian endometrioma diagnosis, especially in women over 50 years of age. Likewise, there is a long history of using TVS for the screening of ovarian tumors [1-5]. However, the usefulness of TVS has not yet been confirmed [6]. There are some reports $[12,13]$ that showed the 
Citation: Kuramoto H (2013) Is Transvaginal Sonograpy Useful in Women's Health Care Programs? Health Care Current Reviews 1: 110. doi: $10.4172 / 2375-4273.1000110$

effectiveness in multimodal trials of co-using biomarkers such as CA125. However, there is no consensus on using the multimodal method of ovarian cancer screening for asymptomatic women $[14,15]$. In contrast, the evaluation of symptomatic patients who are suspected of having ovarian cancer includes TVS and serum CA-125, as well as physical examination [15]. In the present study, the incidence of detecting ovarian tumor with TVS was not significantly increased compared with that of the pelvic exam. TVS, which also has the low specificity for visualizing ovaries, may not have any benefit to screen for ovarian tumors.

Miscellaneous diseases that were found either by TVS or pelvic exam were complementary. Sznto et al. [16] reported that TVS was useful for detecting endometrial hyperplasia when postmenopausal women were screened with TVS. Alcazar [17] mentioned that the endometrium was thicker in hypertensive postmenopausal women. These results suggest that screening for endometrial cancer with TVS may be worthwhile for a selected group of women. Multiple cysts of the cervix may be good information to detect adenomatous malignancy.

\section{References}

1. Van Nagell Jr, Depriest PD, Ueland FR, DeSimone CP, Cooper AL, et al. (2007) Ovarian cancer screening with annual transvaginal sonography: findings of 25,000 women screened. Cancer 109: 1887-1896.

2. Buys SS, Partridge E, Black A, Johnson CC, Lamerato L, et al. (2011) Effect of screening on ovarian cancer mortality: the Prostate, Lung, Colorectal and Ovarian (PLCO) Cancer Screening Randomized Controlled Trial. JAMA 305: 2295-2303.

3. Kobayashi H, Yamada Y, Sado T, Sakata M, Yoshida S, et al. (2008) A randomized study of screening for ovarian cancer: a multicenter study in Japan. Int J Gynecol Cancer 18: 414-420.

4. Menon U, Gentry-Maharaj A, Hallett R, Ryan A, Burnell M, et al. (2009) Sensitivity and specificity of multimodal and ultrasound screening for ovarian cancer, and stage distribution of detected cancers: results of the prevalence screen of the UK Collaborative Trial of Ovarian Cancer Screening (UKCTOCS). Lancet Oncol 10: 327-340.
5. Sharma A, Apostolidou S, Burnell M, Campbell S, Habib M, et al. (2012) Risk of epithelial ovarian cancer in asymptomatic women with ultrasound-detected ovarian masses: a prospective cohort study within the UK collaborative trial of ovarian cancer screening (UKCTOCS). Ultrasound Obstet Gynecol 40: 338344

6. Cambell S (2012) Ovarian cancer: role of ultrasound in preoperative diagnosis and population screening. Ultrasound Obstet Gynecol 40: 245-254.

7. Minoura S (2001) Benign tumors of the ovary. J Jpn Med Assoc 126: S276.

8. Kuramoto $\mathrm{H}$, lida $\mathrm{M}$, Sugimoto $\mathrm{N}$ (2009) Gynecologic screening using cytology, colposcopy and pelvic exam - their efficacy. Yobouigaku 51: 77-83.

9. Hiramoto Y, Nojima S, Touno M, Hataya I, Sasahara J, et al. (2005) Effect of transvaginal sonography in the detection of ovarian lesions at human dock. Ningen dock 20: 24-28.

10. Sato S, Yokoyama Y, Sakamoto T, Futagami M, Saito Y (2000) Usefulness of mass screening for ovarian carcinoma using transvaginal ultrasonography. Cancer 89: 582-588.

11. Kobayashi H, Sumimoto K, Moniwa N, Imai M, Takakura K, et al. (2007) Risk of developing ovarian cancer among women with ovarian endometrioma: a cohort study in Shizuoka, Japan. Int J Gynecol Cancer 17: 37-43.

12. Skates SJ, Menon U, MacDonald N, Rosenthal AN, Oram DH, et al. (2003) Calculation of the risk of ovarian cancer from serial CA-125 values for preclinical detection in postmenopausal women. J Clin Oncol 21: 206-210.

13. Jacobs IJ, Skates SJ, MacDonald N, Menon U, Rosenthal AN, et al. (1999) Screening for ovarian cancer: a pilot randomised controlled trial. Lancet 353: 1207-1210.

14. NIH consensus conference (1995) Ovarian cancer, Screening, treatment, and follow-up. NIH Consensus Panel on Ovarian Cancer. JAMA 273: 491-497.

15. American College of Obstetricians and Gynecologists Committee on Gynecologic Practice (2011) Committee Opinion No. 477: the role of the obstetrician-gynecologist in the early detection of epithelial ovarian cancer Obstet Gynecol 117: 742-746.

16. Sznto F, Horompoli C (1995) The value of transvaginal ultrasonic screening of symptomless postmenopausal women. Orv Hetil 136: 991-993.

17. Alcazar JL (2000) Endometrial sonography findings in asymptomatic hypertensive postmenopausal women. J Clin Ultrasound 28: 175-178. 